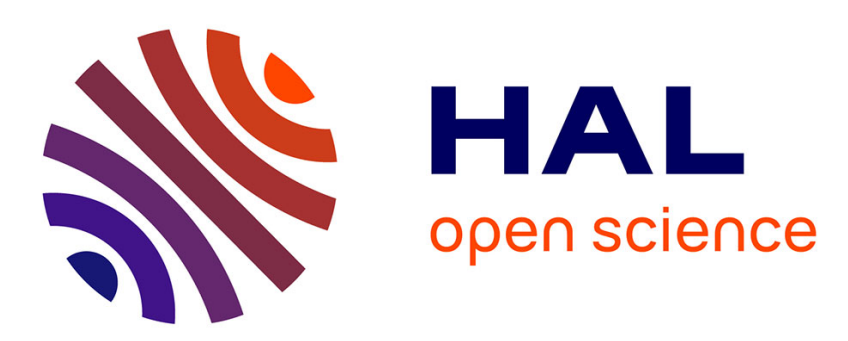

\title{
Electrical Detection of Electron Spin Resonance in Microcrystalline Silicon pin Solar Cells
}

Jan Behrends, Alexander Schnegg, Matthias Fehr, Andreas Lambertz, Stefan Haas, F Finger, Bernd Rech, Klaus Lips

\section{- To cite this version:}

Jan Behrends, Alexander Schnegg, Matthias Fehr, Andreas Lambertz, Stefan Haas, et al.. Electrical Detection of Electron Spin Resonance in Microcrystalline Silicon pin Solar Cells. Philosophical Magazine, 2009, 89 (28-30), pp.2655-2676. 10.1080/14786430903008472 . hal-00519088

\section{HAL Id: hal-00519088 \\ https://hal.science/hal-00519088}

Submitted on 18 Sep 2010

HAL is a multi-disciplinary open access archive for the deposit and dissemination of scientific research documents, whether they are published or not. The documents may come from teaching and research institutions in France or abroad, or from public or private research centers.
L'archive ouverte pluridisciplinaire HAL, est destinée au dépôt et à la diffusion de documents scientifiques de niveau recherche, publiés ou non, émanant des établissements d'enseignement et de recherche français ou étrangers, des laboratoires publics ou privés. 


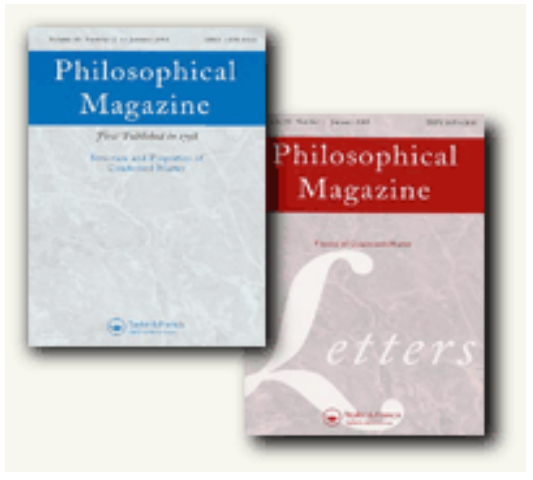

\section{Electrical Detection of Electron Spin Resonance in Microcrystalline Silicon pin Solar Cells}

\begin{tabular}{|c|c|}
\hline Journal: & Philosophical Magazine \& Philosophical Magazine Letters \\
\hline Manuscript ID: & TPHM-08-Dec-0457.R1 \\
\hline Journal Selection: & Philosophical Magazine \\
\hline $\begin{array}{l}\text { Date Submitted by the } \\
\text { Author: }\end{array}$ & 24-Apr-2009 \\
\hline Complete List of Authors: & $\begin{array}{l}\text { Behrends, Jan; Helmholtz-Zentrum Berlin fuer Materialien und } \\
\text { Energie, Abt. Silizium-Photovoltaik } \\
\text { Schnegg, Alexander; Helmholtz-Zentrum Berlin fuer Materialien und } \\
\text { Energie, Abt. Silizium-Photovoltaik } \\
\text { Fehr, Matthias; Helmholtz-Zentrum Berlin fuer Materialien und } \\
\text { Energie, Abt. Silizium-Photovoltaik } \\
\text { Lambertz, Andreas; Forschungszentrum Jülich, Institut für } \\
\text { Energieforschung - Photovoltaik } \\
\text { Haas, Stefan; Forschungszentrum Jülich, Institut für } \\
\text { Energieforschung - Photovoltaik } \\
\text { Finger, F; Forschungszentrum Jülich, IEF5-PV } \\
\text { Rech, Bernd; Helmholtz-Zentrum Berlin fuer Materialien und } \\
\text { Energie, Abt. Silizium-Photovoltaik } \\
\text { Lips, Klaus; Helmholtz-Zentrum Berlin fuer Materialien und Energie, } \\
\text { Abt. Silizium-Photovoltaik }\end{array}$ \\
\hline Keywords: & conductivity, defects, EPR, microcrystalline silicon, photovoltaics \\
\hline Keywords (user supplied): & solar cells, electron spin resonance \\
\hline \multicolumn{2}{|c|}{$\begin{array}{l}\text { Note: The following files were submitted by the author for peer review, but cannot be converted } \\
\text { to PDF. You must view these files (e.g. movies) online. }\end{array}$} \\
\hline TPHM_08_Dec_0457_revise & tex \\
\hline
\end{tabular}




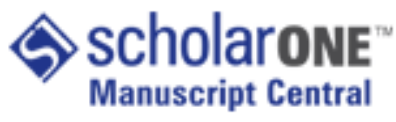




\title{
RESEARCH ARTICLE
}

\section{Electrical Detection of Electron Spin Resonance in Microcrystalline Silicon pin Solar Cells}

\author{
J. Behrends ${ }^{\dagger *}$, A. Schnegg ${ }^{\dagger}$, M. Fehr ${ }^{\dagger}$, A. Lambertz ${ }^{\ddagger}$, S. Haas ${ }^{\ddagger}$, F. Finger ${ }^{\ddagger}$, B. Rech ${ }^{\dagger}$, K. \\ $\operatorname{Lips}^{\dagger}$ \\ ${ }^{\dagger}$ Helmholtz-Zentrum Berlin für Materialien und Energie (formerly \\ Hahn-Meitner-Institut), Institut für Silizium-Photovoltaik, Kekuléstr. 5, D-12489 Berlin, \\ Germany \\ ${ }^{\ddagger}$ Forschungszentrum Jülich, Institut für Energieforschung — Photovoltaik, D-52425 \\ Jülich, Germany
}

(April 24, 2009)

\begin{abstract}
Pulsed electrically detected magnetic resonance (pEDMR) was employed to study spindependent processes that influence charge transport in microcrystalline $(\mu \mathrm{c}-\mathrm{Si}: \mathrm{H})$ pin solar cells. Special emphasis was put on the identification of the signals with respect to the individual layers of the cell structure. For this to achieve, we systematically modulated the morphology of the highly doped n- and p-layers from amorphous to microcrystalline. By combining the information obtained from low-temperature $(T=10 \mathrm{~K})$ pEDMR spectra and from the deconvoluted time evolution of spectrally overlapping resonances, we found signals from conduction band tail states as well as phosphorus donor states in samples containing an amorphous n-type layer and a resonance associated with valence band tail states in samples with amorphous p-layer. Moreover, several signals from the intrinsic microcrystalline absorber layers could be identified. An additional resonance at $g=1.9675(5)$, which has not been observed in EDMR before, was found. We assign this signal to shallow donors in the Al-doped $\mathrm{ZnO}$ layer which is commonly used as transparent conducting oxide in thin-film solar cells. The experimental findings are discussed in the light of various spin-dependent transport mechanisms known to occur in the respective layers of the pin structure.
\end{abstract}

Keywords: solar cells; photovoltaics; conductivity; defects; microcrystalline silicon; electron spin resonance

\section{Introduction}

Today's solar cell technology is mainly based on crystalline silicon (c-Si) wafers. The high efficiencies that can be reached with wafer-based technologies, however, come along with the energy and cost intensive wafer production process. In contrast to that, solar cells made from hydrogenated amorphous silicon (a-Si:H) and its microcrystalline counterpart ( $\mu \mathrm{c}-\mathrm{Si}: \mathrm{H})$ can be deposited directly on inexpensive substrates like glass and have the potential to be superior with regard to material consumption and cost effectiveness. The production of these thin-film devices, which are usually realised in a pin configuration, was made possible by the pioneering discovery by W.E. Spear and P.G. LeComber in 1975 that amorphous silicon can be doped during deposition [1]. Unfortunately, a-Si:H and $\mu \mathrm{c}-\mathrm{Si}: \mathrm{H}$ suffer from

\footnotetext{
*Corresponding author. Email: jan.behrends@helmholtz-berlin.de
} 
an inferior electronic quality compared to crystalline silicon. This is due to defect states in the band gap of the material resulting from silicon dangling bonds $(\mathrm{db})$ as well as band tail states close to the band edges. In thin-film solar cells, trapping and recombination at localised defects are the main limiting factors for the cell performance. Due to the fact that these defects are often paramagnetic (in the case of $\mathrm{db}$ ) or can be made paramagnetic by light excitation (in the case of tail states), electron spin resonance (ESR) can reveal quantitative as well as structural information [2-4]. For instance, ESR on powder samples is routinely being used to optimise the deposition parameters for a-Si:H and $\mu \mathrm{c}-\mathrm{Si}: \mathrm{H}$ growth [5]. Although a correlation between material quality as determined by ESR and efficiency of resulting solar cells is well established, it still remains questionable if the defect properties of the thin-films in the device and in powder samples are indeed identical, since the boundary conditions for the layer growth are different. Attempts to extend ESR studies to fully processed thin-film solar cells are restricted by two major limitations. Firstly, the detection sensitivity of conventional X-band ESR is typically too low for the spin concentrations found in state-of-the-art thin-film solar cells, and secondly, paramagnetic centres in contact layers and the substrate may introduce additional ESR signals, which do not influence the solar cell efficiency. To overcome the limitations of conventional ESR, we applied electrically detected magnetic resonance (EDMR) (for a review see [6] and references therein). This technique allows the investigation of paramagnetic states influencing transport and recombination through spin-dependent processes in thin films [7-9] and devices [10, 11] at ultra high sensitivity $[12,13]$. Since EDMR is based on photocurrent measurements, it is inherently only sensitive to defects influencing the conductivity of the solar cell and therefore its performance and efficiency. The uniqueness of EDMR originates from its capability to directly relate the microscopic properties of current limiting paramagnetic states with macroscopic transport in the device. Probing the sample properties by spin interactions, defect states may be assigned by their position in the EDMR spectrum. Further on, EDMR line shape analysis yields highly desired information about the microscopic defects. Though conventional cwEDMR proved to provide valuable information about defect states in the material, a complete picture of the transport mechanisms in the solar cell may only be obtained by time-resolved EDMR techniques. Employing state-of-the-art pulsed ESR instrumentation in combination with sensitive current detection, we recently succeeded in the development of several pulsed (p)EDMR detection schemes providing 2D resonance field vs. evolution time EDMR spectra [14-16]. Extending the capabilities of EDMR into time domain, pEDMR allows assigning defect states to complex transport mechanisms.

In this paper, we exploit pEDMR to identify paramagnetic centres in $\mu \mathrm{c}-\mathrm{Si}: \mathrm{H}$ solar cells. Despite its capabilities, pEDMR spectra from samples containing many layers of different material typically contain overlapping signals, which may only be identified by combining appropriate $\mathrm{pEDMR}$ techniques with a systematic variation of the sample morphology. While the types of localised defect states that are present in either $\mu \mathrm{c}-\mathrm{Si}: \mathrm{H}$ or a-Si:H are similar, the fingerprints of their respective ESR signals (most importantly the $g$ value) show significant differences between both morphologies [6]. All measurements were performed at $T=10 \mathrm{~K}$ in order to benefit from the enhanced signal-to-noise ratio at low temperatures which is indispensable for a thorough analysis of the EDMR signals and the assignment to the respective defect states. However, it is worthwhile noting that mechanisms that govern the spin-dependent transport at $T=10 \mathrm{~K}$ differ significantly from the performancelimiting processes at normal solar cell operating conditions. Nevertheless, this study constitutes the starting point for prospective investigations of charge transport at 


\section{Samples}

All $\mu \mathrm{c}-\mathrm{Si}: \mathrm{H}$ pin solar cells investigated in this study are based on the layer sequence shown in figure 1. The samples were deposited on quartz substrates using plasma enhanced chemical vapour deposition (PECVD) at the Forschungszentrum Jülich with a process that was shown to reach efficiencies above $10 \%$ on areas of $1 \mathrm{~cm}^{2}$ [17]. Four series of solar cells were prepared, where the p- (boron) and ndoped (phosphorus) layers were either composed of amorphous or microcrystalline silicon keeping the deposition conditions for the intrinsic absorber layer unchanged. The solar cells were grown on Al-doped $\mathrm{ZnO}$, always starting with the thin p-doped silicon layer. A $\mathrm{ZnO} / \mathrm{Al}$ layer stack was used as back reflector and contact. Note that the chosen cell structure was not optimised for high solar cell conversion efficiency but was solely designed for the purpose to reliably study the influence of the various contact layers on the EDMR behaviour.

To discriminate between $\mathrm{pEDMR}$ signals arising from the intrinsic $\mu \mathrm{c}-\mathrm{Si}: \mathrm{H}$ absorber or the highly doped layers, four samples with the layer sequence described in table 1 were prepared. It should be noted here that different thicknesses of the $\mathrm{ZnO}$ layer were used for the four samples. However, we believe that this does not affect the pEDMR signals originating from the silicon pin structures. Throughout this article the sample structures will be referred to as sample A, B, C, and D. Schematic energy-band diagrams of all cells neglecting the $\mathrm{ZnO}$ as well as metal layers are depicted in figure 2 .

For the pEDMR samples a special contact structure consisting of $50 \mathrm{~mm}$ long, $0.5 \mathrm{~mm}$ wide and $100 \mathrm{~nm}$ thin $\mathrm{Ag}$ strip lines was used to establish an electrical connection to contact pads that are positioned outside the microwave (mw) res- 


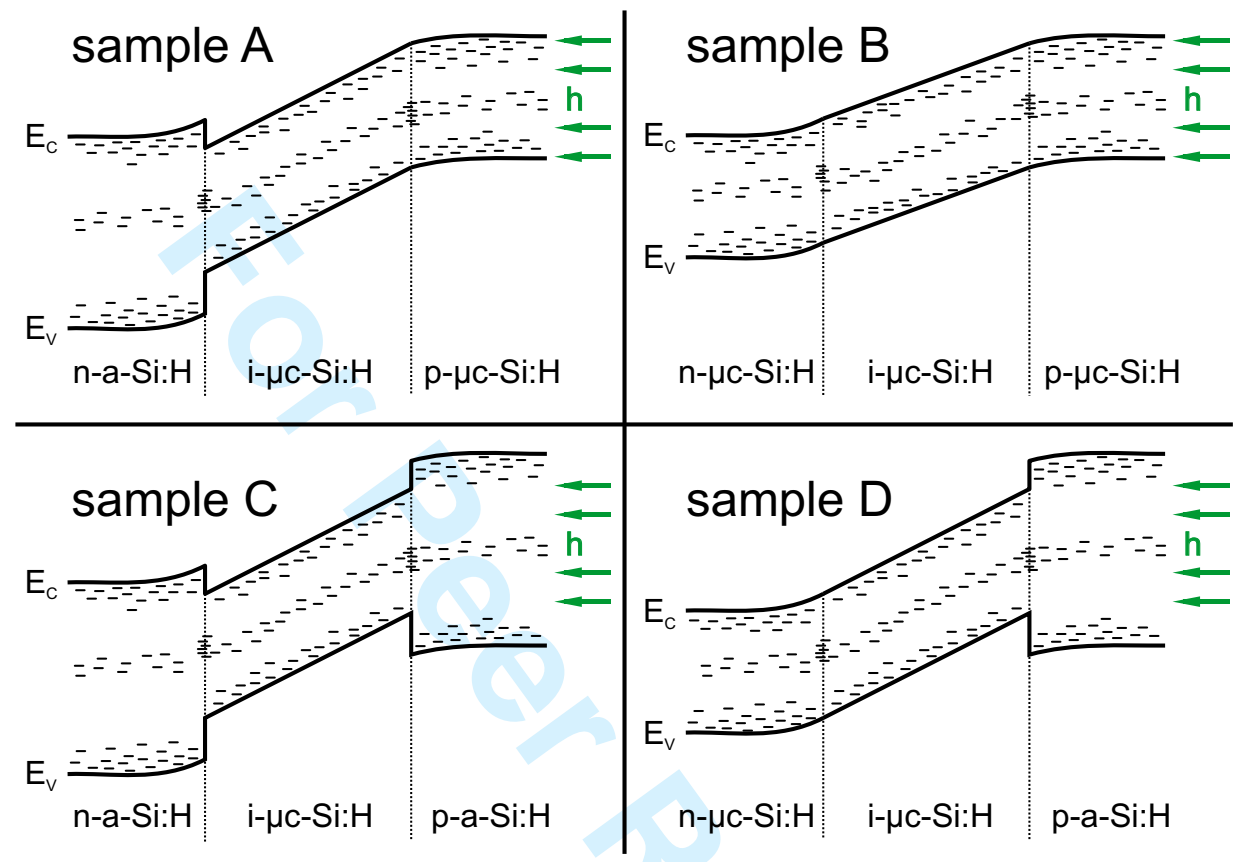

Figure 2. Schematic representation of the energy-band diagrams at room temperature without illumination for samples A to D. The morphology of the doped layers can be identified by the different band gaps. Localised defect states are sketched for all layers without taking into account the difference in their density of states with respect to energy, doping concentration and layer morphology. Note that the ZnO and metal layers have been omitted. In all measurements presented here, the cells were illuminated through the substrate (p-side of the pin structure).

onator. Through this design the perturbation of the eigenmodes of the dielectric mw resonator by the electric circuitry is minimized. Details of the contact scheme and its influence on the pEDMR measurement can be found elsewhere [18]. The patterning of the contacts as well as the confinement of the active solar cell area to $1 \mathrm{~mm}^{2}$ was realised by laser scribing techniques. Figure 1 depicts a photograph of a completely processed sample, clearly showing the structure of the EDMR samples and the contact pads opposite to the solar cell.

\section{Experimental details}

The pEDMR setup is based on a commercial X-band ESR spectrometer Bruker Elexsys E580 which was upgraded by the equipment for electrical detection. The sample is mounted in a dielectric resonator and cooled using a continuous flow helium cryostat with optical access. All EDMR measurements described in the following were carried out at $T=10 \mathrm{~K}$ under illumination through the quartz substrate with a cold light source at approximately $50 \mathrm{~mW} \mathrm{~cm}^{-2}$. Figure 3 shows current voltage curves obtained under these experimental conditions. Note that the low-temperature $I-V$ curves differ significantly from those one would obtain at 


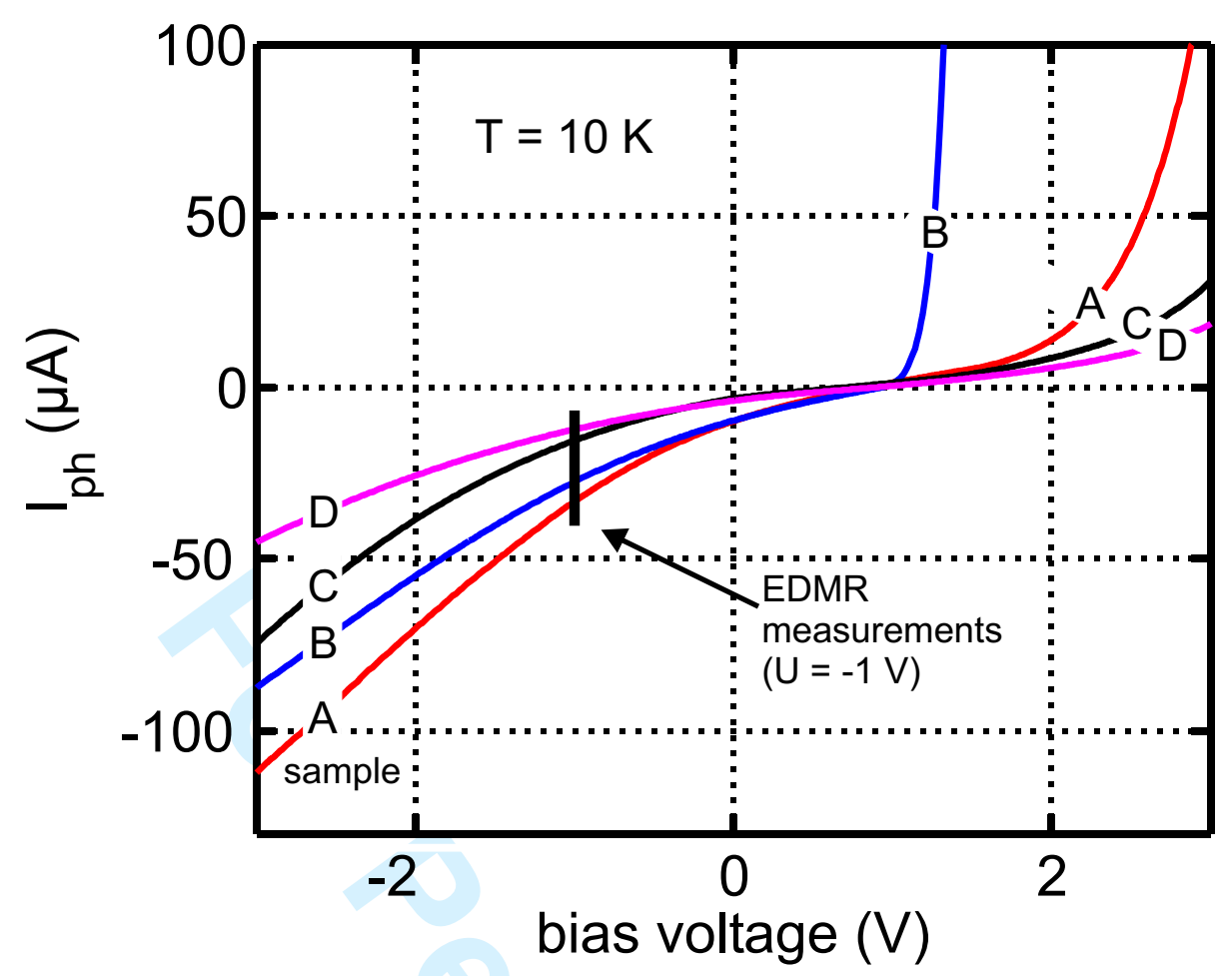

Figure 3. Low-temperature current-voltage characteristics of samples A to D under illumination $\left(50 \mathrm{~mW} \mathrm{~cm}^{-2}\right)$. All EDMR measurements were carried out at a constant voltage $U=-1 \mathrm{~V}$ (reverse bias regime of the solar cell). This point is indicated in the plot by a vertical line.

room temperature. At positive voltages this can primarily be attributed to barriers — in particular the heterobarrier between a-Si:H and $\mu \mathrm{c}-\mathrm{Si}: \mathrm{H}$ - which hinder the charge transport in the device. This is in line with the observation that the $I-V$ curve of sample B ( $\mu \mathrm{c}-\mathrm{Si}: \mathrm{H} \mathrm{n}$ - and p-layers) varies markedly from the other curves in the forward bias regime.

For the EDMR measurements, a battery-based constant voltage source (Stanford Research SIM928) was used to apply a reverse bias voltage of $U=-1 \mathrm{~V}$ to the sample. Due to the different current-voltage-characteristics of the respective samples A to D (cf. figure 3), this corresponds to photocurrent densities between -1.3 and $-3.3 \mathrm{~mA} \mathrm{~cm}^{-2}$. At this voltage the qualitative behaviour of the illuminated $I-V$ curves was the same for all samples, and in none of the samples the saturation regime of carrier extraction was reached. We believe that at $U=-1 \mathrm{~V}$ the current extraction mechanism is the same for all specimens under study and is dominated by drift in the electric field of the intrinsic $\mu \mathrm{c}-\mathrm{Si}: \mathrm{H}$ layer.

Two-dimensional mappings of the spin-dependent processes were obtained by recording the transient photocurrent changes $\Delta\left|I_{\mathrm{ph}}\right|$ following a mw pulse excitation as a function of the external magnetic field. While the mw pulses have a length of typically several 10 to a few $100 \mathrm{~ns}$, the detection of $\Delta\left|I_{\mathrm{ph}}\right|$ is carried out for some $100 \mu \mathrm{s}$. We used a current/voltage converter (Elektronik-Manufaktur Mahlsdorf) that was especially designed for transient photocurrent detection in pEDMR measurements and that allows for a good trade-off between bandwidth and noise. The EDMR setup is schematically shown in figure 4. This instrument consists of three main components as indicated in figure 4 . In the transimpedance amplifier the current signal is converted into a voltage which is then filtered by a bandpass with lower cut-off frequency of $1 \mathrm{~Hz}$ to remove the DC-component of $I_{\mathrm{ph}}$. The upper cut-off frequency can be varied in order to control the time resolution of the detection setup. The resulting signal is then amplified to a level that is suitable 
Figure 4. Sketch of the pEDMR setup based on a commercial Bruker Elexsys E580 X-band ESR spectrometer. The sample is located in a microwave resonator which is placed in a He-cryostat with a window for optical access. A constant voltage source is used to apply a voltage $U_{S}$ to the sample and thus establishing a steady-state photocurrent $I_{\mathrm{ph}}$. Changes of the photocurrent, $\Delta\left|I_{\mathrm{ph}}\right|$, are recorded as a function of time using a current detection unit shown in the lower part. The equivalent circuitry of the solar cell can be considered as a parallel circuit consisting of current source, resistor, and capacitor. Thus, it inherently acts as a lowpass filter for changes of the photocurrent and therefore limits the time resolution. The signal $\Delta\left|I_{\mathrm{ph}}\right|$ is converted into a voltage, filtered, and amplified to a level that is sufficient for the transient recorder of the ESR-spectrometer.

for the transient recorder (Bruker SpecJet).

The time evolution of the photocurrent after mw excitation (in the following referred to as pEDMR transient) is determined by the rate coefficients of the spindependent microscopic processes as described in refs. [14, 18]. However, it is generally difficult to determine these coefficients directly from the pEDMR transients since $R C$ time constants of the detection electronics or the dielectric relaxation times of the sample dominate the experimentally obtained pEDMR transients. To demonstrate this, figure 5 shows two pEDMR transients of sample A that were obtained using two different current/voltage converters under otherwise identical conditions. The transient labelled with 'SR570' was measured using a Stanford Research SR570 current amplifier which is often used for pEDMR. The transient labelled 'EMM' was recorded with the current/voltage converter described above (Elektronik-Manufaktur Mahlsdorf) with the bandwidth set to a value corresponding to a rise time of $2 \mu \mathrm{s}$. Both transients clearly exhibit markedly different dynamics, although the experimental conditions were the same. The rise and fall times of the 'SR570'-transient are slower and the sign reversal of the transient is shifted to longer times. This difference is simply due to the lowpass filtering by the SR570 current amplifier. This can be demonstrated by numerical lowpass filtering of the 'EMM' data with the response time of the SR570 amplifier taking into account the $R C$ equivalent circuitry as indicated in figure 4 . As shown in figure 5 , the filtered 'EMM' data becomes identical with that of 'SR570'. To elucidate the influence of the sample (not necessarily of the microscopic processes) on the transients, it can be helpful to model the pEDMR transients. Assuming that the transients are determined by two exponential functions with two time constants linked to the spin-dependent process under observation and further taking into account the $R C$ time constants induced by the current detection setup and the sample itself, good 
Figure 5. pEDMR transients obtained from sample A. Two different current amplifiers as described in the text were used in order to demonstrate their influence on the transient dynamics. The curve labelled with 'EMM filtered' was generated by applying a numerical lowpass filter to the data of 'EMM'. The curve 'EMM fit' represents a least square fit of a model function consisting of two exponential functions (time constants $\tau_{1}$ and $\tau_{2}$ ) and taking into account lowpass filtering (time constant $\tau_{R C}$ ) to the experimental data 'EMM' (fit results: $\tau_{1}=0.75 \mu \mathrm{s}, \tau_{2}=65 \mu \mathrm{s}, \tau_{R C}=4 \mu \mathrm{s}$ ). The inset shows an enlarged view of the first $20 \mu$ s of the transients.

fits to the experimentally obtained data can be achieved. This is demonstrated in figure 5 for the unfiltered 'EMM'-transient. Regardless of the strong influence of the detection electronics on the pEDMR dynamics, the pEDMR dynamics can be used to deconvolute spectrally overlapping signals as will be shown in this article.

The geometry of solar cells to be analysed by pEDMR is inherently limited by the dimensions of the mw resonator which in turn is determined by the wavelength of the microwave. For X-band ( $\nu \approx 10 \mathrm{GHz}$, wavelength $3 \mathrm{~cm}$ ), a solar cell area of $1 \times 1 \mathrm{~mm}^{2}$ (cf. figure 1 ) has proved to be reasonably small to yield a homogeneous mw amplitude over the whole active area of the solar cell. We have carefully checked that defects induced by the preparation and processing — in particular defects located at the edges — do not affect the experiments by comparing results obtained from cells with different geometries. This is particularly important when studying coherent effects by means of pEDMR. Since the active area of solar cells studied with EDMR is rather small, additional defects induced by the preparation and processing - especially defects located at the edges - can significantly affect the experiments. To exclude that the EDMR spectra are dominated by such defects, we prepared sample D with two different solar cell geometries with clearly varying edge length as shown by the optical micrographs in figure $X$. The respective pEDMR spectra, i.e. $\Delta\left|I_{\mathrm{ph}}\right|$ as a function of magnetic field, are shown in figure $\mathrm{X}$. Within the accuracy of the measurement, the normalised spectra are identical, indicating that the same underlying microscopic processes are involved. It is therefore safe to assume that no new types of defects are introduced by the specific processing of the solar cells for pEDMR measurements. The signal-to-noise ratio is significantly reduced for the $2 \times 0.5 \mathrm{~mm}^{2}$ sample which is attributed to the smaller photocurrent (and consequently also smaller $\Delta\left|I_{\mathrm{ph}}\right|$ ) resulting from the fact that the illumination 


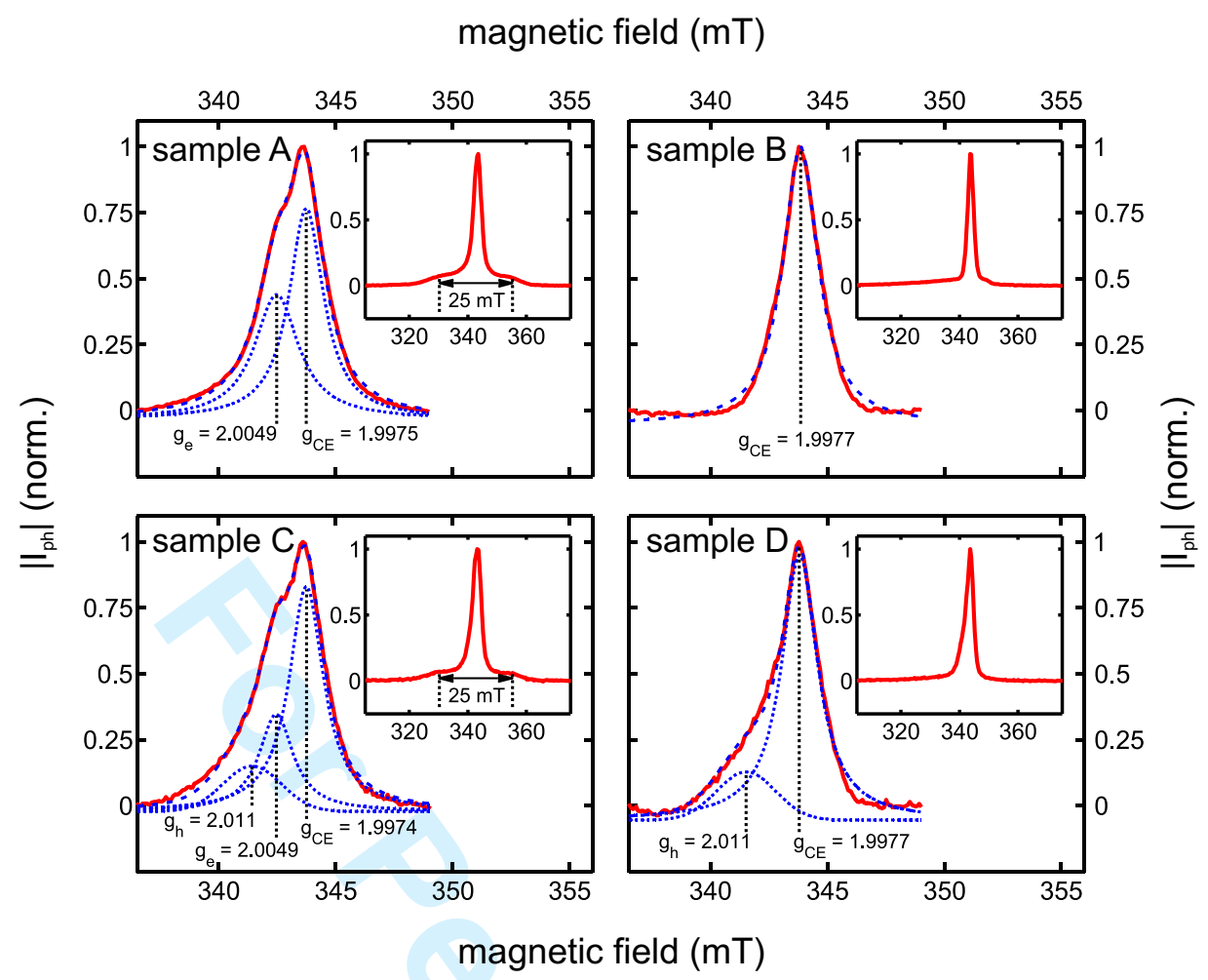

Figure 6. pEDMR spectra of samples A to D taken at $t=2.2 \mu$ s after the mw excitation at $T=10 \mathrm{~K}$ using a pulse length $\tau$ of 320 ns (solid line: experiment; dashed line: fit to the experimental data; dotted lines: individual spectral components as obtained from the fit). The resonance positions are indicated by vertical dotted lines. The insets show spectra on a broader magnetic field range measured under the same experimental conditions except for a mw power increased by $9 \mathrm{~dB}$ in order to enhance the broad spectral components. In addition, $\tau$ was set to values between 50 and $60 \mathrm{~ns}$, which in all cases corresponds to a spin flip angle of $\phi=\Omega \cdot \tau=\pi$, with $\Omega$ being the Rabi frequency.

eondition was optimised for a square-shaped active area. All following experiments have been carried out on square-shaped solar cells.

\section{Results and discussion}

The pEDMR datasets obtained from samples A to D differ with respect to both their spectral shape as well as their transient behaviour. In the following, we will first identify the defect states by their resonance position in the pEDMR spectra. Combining these pieces of information with the dynamics and Rabi oscillations of the respective spectral components, we will, in a second step, conclude on the underlying transport and recombination mechanisms.

\subsection{Identification of defect states}

Figure 6 shows the pEDMR spectra of samples A to D recorded at $t=2.2 \mu$ s after applying the mw pulse. The insets display spectra taken from the same samples on a broader magnetic field range. Common to all samples is that they contain a narrow component centred around the $g$ value of the free electron and, in addition, a broad $(>20 \mathrm{mT}$ ) component with lower intensity. Beside these similarities, further inspection of the spectra unravels that the narrow component as well as the broad line consist of several EDMR signals which, in addition, differ from sample to sample.

For the identification of the defects contributing to the pEDMR spectra, we 
analysed the resonances by fitting Lorentzian and Gaussian functions to the narrow spectra. The line shapes (Gaussian or Lorentzian) were chosen to achieve best fits to the experimental data. Note that the mw power in pEDMR is considerably higher than in cwEDMR. As a result, mw power broadening of the lines can lead to deviations between line shapes and line widths obtained from $\mathrm{cw}-$ and $\mathrm{pEDMR}$ experiments. This applies especially to narrow lines. The results of the least square fits can be summarised as follows: Best fits to the experimental spectra can be obtained when assuming a resonance at $g_{\mathrm{CE}}=1.9975(5)$ for all samples. Additionally, we find a signal at $g_{\mathrm{e}}=2.0049(5)$ in samples A and C (amorphous n-layer) as well as a line at $g_{\mathrm{h}}=2.011(1)$ in samples $\mathrm{C}$ and $\mathrm{D}$ (amorphous p-layer).

The broad spectra reveal an additional pair of lines separated by $25 \mathrm{mT}$, indicating the presence of hyperfine interaction in samples $\mathrm{A}$ and $\mathrm{C}$ (amorphous n-layer). Both lines are symmetric to $g_{\mathrm{P}} \approx 2.003$. This feature is absent in samples B and D (microcrystalline n-layer). Instead, in samples B and D an asymmetric broad signal is uncovered. For an unambiguous assignment of the microscopic origin of this line we will discuss its spectral shape in more detail below. In the following, we will assign the resonance positions to defect states in the samples based on findings from previous EDMR and ESR studies on thin films as well as powder samples.

\subsubsection{Intrinsic $\mu c-S i: H$}

In intrinsic and n-doped $\mu \mathrm{c}-\mathrm{Si}: \mathrm{H}$ an ESR resonance at $g=1.997-1.998$ is reported in various studies which is associated with shallow localised states in energetic proximity to the conduction band, typically referred to as CE states $[2$, 19-23]. It was demonstrated by cw- and pEDMR measurements on $\mu \mathrm{c}-\mathrm{Si}: \mathrm{H}$ films that these states are involved in hopping transport at low temperatures as well as in tunnelling recombination between $\mathrm{CE}$ and dangling bond states [3, 22]. Hence, we assign the signal at $g_{\mathrm{CE}}=1.9975(5)$, that was observed in all samples, to originate from $\mathrm{CE}$ states in the intrinsic microcrystalline absorber layer.

\subsection{2. n-doped a-Si:H}

Both samples that contain an a-Si:H n-layer (samples A and C) show signals at $g_{\mathrm{e}}=2.0049(5)$ and $g_{\mathrm{P}} \approx 2.003$, which are absent in samples B and D. This is a strong indication that these resonances are related to localised states in n-a-Si:H. In the case of line at $g_{\mathrm{P}}$ (in the following referred to as 'P signal'), this interpretation is supported by the fact that the EDMR signal consists of a pair of Gaussian lines with equal intensity that are split by $25 \mathrm{mT}$. This splitting is well known to arise from hyperfine (hf) interaction between phosphorus electron and nuclear spins in amorphous silicon [24] and was shown to also exist in the cw- and pEDMR spectra of phosphorus-doped a-Si:H $[25,26]$. It is worthwhile noting here that phosphorus is only incorporated in the thin n-doped layer and that for phosphorus-doped $\mu \mathrm{c}-\mathrm{Si}: \mathrm{H}$ neither the $4.2 \mathrm{mT}$ hyperfine splitting known from c-Si nor the abovementioned $25 \mathrm{mT}$ split hf lines are observed $[2,27]$. This is consistent with our observations. The signal at $g_{\mathrm{e}}$ is presumably associated with conduction band tail states in the $\mathrm{n}$-a-Si:H layer and is also observed in $\mathrm{n}-\mathrm{a}-\mathrm{Si}: \mathrm{H} / \mathrm{c}-\mathrm{Si}$ solar cells [26]. EDMR investigations at temperatures between 100 and $150 \mathrm{~K}$ have revealed that a signal at $g=2.0044$ may be associated with hopping of electrons among band tail states in a-Si:H (known as 'e signal') [25, 28]. However, LESR measurements have shown an increase of the $g$ value with decreasing temperature [29]. Thus, the discrepancy between $g=2.0044$ and $g_{\mathrm{e}}=2.0049(5)$ - as it was found in our measurements at $T=10 \mathrm{~K}$ - might be explained by the temperature difference. This is also in line with low-temperature pEDMR results for hopping via a-Si:H tail states in a-Si:H/c-Si solar cells $[18,26]$. 


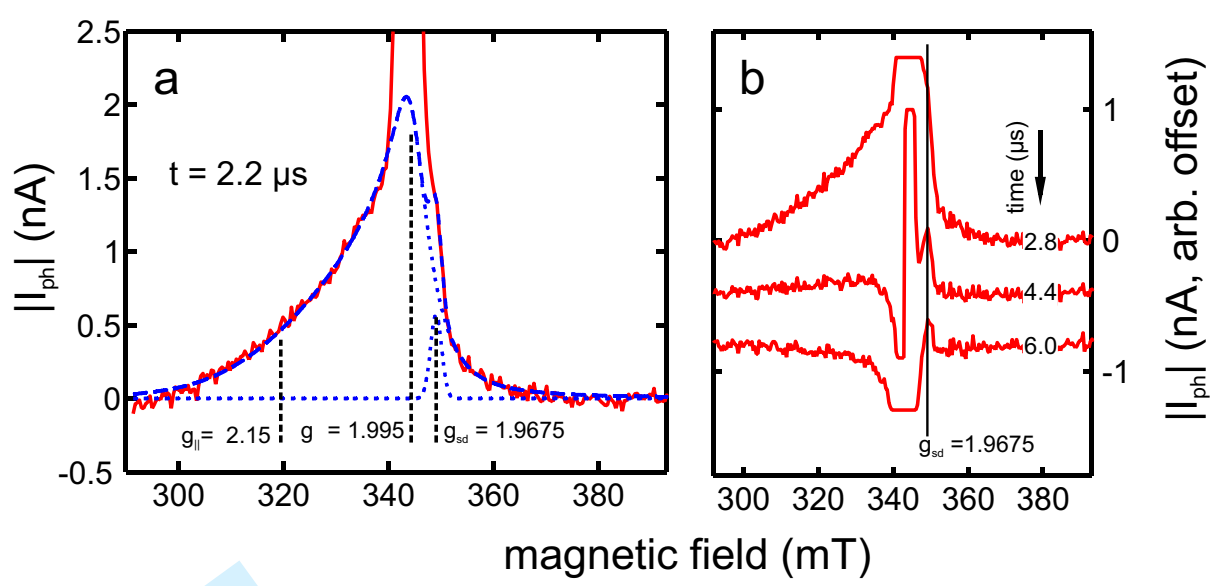

Figure 7. Asymmetric line that was found in samples B and D ( $\mu$ c-Si:H n-layer). (a) Enlarged view of the pEDMR spectrum of sample B as shown in figure 6 with an ordinate range covering the first $10 \%$ of the maximum signal amplitude (solid line: experiment; dashed line: results of a simulation consisting of an inhomogeneously broadened line with parameters given in the text and a symmetric Gaussian line at $g_{\text {sd }}=1.9675(5)$; dotted lines: both spectral components separately). (b) pEDMR spectra of sample B for several delays after the mw excitation. The solid vertical line serves as a $g$ value marker. Note that the time behaviour of the asymmetric line and the sd resonance is considerably different.

\subsection{3. p-doped $a$-Si:H}

Analogous to electron hopping described before, hopping of holes via valence band tail states in boron-doped a-Si:H can also contribute to the photocurrent. The corresponding resonance (' $\mathrm{h}$ signal') has been investigated in the past using EDMR $[25,28,30]$ and optically detected magnetic resonance (ODMR) revealing $g=2.011$ to 2.013 [31-33]. In the solar cells C and D (a-Si:H p-layer) we found a signal at $g_{\mathrm{h}}=2.011$ which we assign to valence band tail states in a-Si:H. No evidence of boron acceptor states could be found.

\subsubsection{The $\mathrm{CH}$ signal}

In the pEDMR spectra of samples B and D ( $\mu \mathrm{c}-\mathrm{Si}: \mathrm{H}$ n-layer) the $25 \mathrm{mT}$ splitting of the phosphorous signal is absent. Further inspection of these spectra unravels an asymmetric signal of different shape (see figure 6). Figure 7a gives an enlarged view of the pEDMR spectrum of sample B with a range covering the first $10 \%$ of the ordinate range of the plot in figure 6 . The line shape of the resonance resembles the powder pattern of an asymmetric $g$ tensor. The dashed curve in figure $7 \mathrm{a}$ depicts the results of a simulation (simulation program EasySpin [34], simulation parameters: $g_{\|}=2.15, g_{\perp}=1.995$, field-dependent inhomogeneous broadening $\left.\Delta g_{\|}=0.2, \Delta g_{\perp}=0.01\right)$. Note that field-dependent inhomogeneous broadening has been reported in many cases concerning disorder in amorphous silicon $[4,35,36]$. Asymmetric broad lines in echo-detected field-sweep spectra obtained by pulsed ESR have been reported in literature to be associated with holes in localised valence band tail states (referred to as CH signal) in undoped and boron doped $\mu \mathrm{c}-\mathrm{Si}: \mathrm{H}$ [27]. Therefore, it seems to be justified to assign the asymmetric signal described above to $\mathrm{CH}$ states in the intrinsic absorber layer which is present in all samples. The $\mathrm{CH}$ signal is indeed visible in the spectra of all samples, however, in samples A and $\mathrm{C}$ it is almost completely hidden underneath the high-field hf-satellite which has a higher intensity. We can exclude that this line originates from the a-Si:H layers because the ESR signature from valence band tail states in a-Si:H is different (cf. h signal described before) [30]. It should be noted that it is complicated to resolve broad lines in cwEDMR. In pulsed ESR and EDMR, however, one benefits from a flat baseline which facilitates the observation of broad spectral components. 


\subsubsection{The sd signal}

Further examination of figure 7 a reveals an additional spectral component with Gaussian line shape at $g_{\mathrm{sd}}=1.9675(5)(\mathrm{FWHM}=2.5 \mathrm{mT})$ which is represented by a dotted curve in figure 7a. The fact that this line (referred to as 'sd signal') is not part of the asymmetric resonance becomes clear when evaluating the time evolution of the pEDMR spectra. This is illustrated in figure $7 \mathrm{~b}$ where three spectra taken between 2.8 and $6.0 \mu$ s after the mw excitation are shown. The position of $g_{\text {sd }}$ is marked by a solid vertical line. The central lines have been cut in order to facilitate the analysis of the weaker signals. The plot unambiguously shows that the sd signal has a distinctively different time dependence as compared to the asymmetric line, indicating that both signals arise from different paramagnetic centres and microscopic processes. While the asymmetric line having qualitatively identical shape was found in all four samples, the sd signal could exclusively be observed in sample B.

To the best of our knowledge, no ESR or EDMR signals caused by paramagnetic states at $g_{\mathrm{sd}}=1.9675(5)$ have been reported for either a-Si:H or $\mu \mathrm{c}-\mathrm{Si}: \mathrm{H}$. However, resonances at similar $g$ values were obtained from shallow donors in $\mathrm{ZnO}$ [37, 38]. Here, the observed $g$ value critically depends on the morphology (crystallite size) of the respective $\mathrm{ZnO}$ material. The photogenerated charge in all solar cells investigated traverses the $\mathrm{ZnO}$ layers on both sides of the pin structure which are both degenerately doped with aluminium. We therefore assign the sd signal to originate from either of the $\mathrm{ZnO}$ layers. Its $g$ value and the fact that this resonance is only observed in sample B suggests that it is connected to a shallow donor state resulting from $\mathrm{Al}$ incorporated in the $\mathrm{ZnO}$ layer on the $\mathrm{p}-\mu \mathrm{c}-\mathrm{Si}: \mathrm{H}$ side or at the interface between these two layers. From this argumentation, the sd resonance should also appear in sample A, but here the line may be buried below the broad and rather intensive hf satellites. We can exclude that the resonance is connected to transport at or through the $\mathrm{ZnO} / \mathrm{n}-\mu \mathrm{c}-\mathrm{Si}: \mathrm{H}$ interface since the sd signal is not visible in the spectrum of sample $\mathrm{D}$.

In summary, the line parameters of the individual signals and the associated paramagnetic defects in the respective layers are listed in table 2.

\subsection{Assignment to microscopic transport and recombination mechanisms}

In order to fully describe the involvement of different defect states in charge transport processes, information obtained from line shape analyses have to be combined with the information extractable from pEDMR transients and Rabi oscillations.

In general, EDMR signals are due to changes in the probability for spindependent transitions between paramagnetic states. All signals observed in this study can be explained within the framework of a spin pair model in which the transition probability depends on the relative spin orientation of both spin partners of the pair [14, 39, 40]. Upon application of a strong mw pulse which is in resonance with either of the participating states, the transition probability generally increases, leading to an increase of the corresponding transition rate. However, this rate change of a microscopic process can influence the macroscopic photocurrent of a pin $\mu \mathrm{c}-\mathrm{Si}: \mathrm{H}$ solar cell in different ways, depending in a complicated way on the microscopic mechanism, the device physics, and, in the particular case of pin solar cells, on the bias voltage applied to the sample $[41,42]$.

In the present case all experimental data has been obtained in the reverse bias regime $(U=-1 \mathrm{~V})$ where the photocurrent is negative. In this situation, the pEDMR transient exhibits a relative enhancement of the photocurrent $\left(\Delta\left|I_{\mathrm{ph}}\right|>0\right)$ immediately after the mw pulse for a spin-dependent hopping process, implying an improvement of carrier extraction due to the selective mw excitation. The resonant 
Table 2. $g$ values, line shapes and line widths obtained from least-square fits to the pEDMR spectra with narrow and wide magnetic field range (see figure 6). Details are given in the text.

\begin{tabular}{|c|c|c|c|c|c|c|}
\hline & & sample A & sample B & sample C & sample D & $\begin{array}{l}\text { paramagnetic } \\
\text { state }\end{array}$ \\
\hline $\mathrm{CE}$ & $\begin{array}{l}g_{\mathrm{CE}}(\text { Lorentzian }) \\
\mathrm{FWHM}_{\mathrm{CE}}(\mathrm{mT})\end{array}$ & $\begin{array}{l}1.9975(5) \\
1.8(1)\end{array}$ & $\begin{array}{l}1.9977(5) \\
1.8(1)\end{array}$ & $\begin{array}{l}1.9974(5) \\
1.8(1)\end{array}$ & $\begin{array}{l}1.9977(5) \\
1.9(1)\end{array}$ & $\begin{array}{l}\text { cond. band tail } \\
\mu \mathrm{c}-\mathrm{Si}: \mathrm{H}\end{array}$ \\
\hline $\mathrm{e}$ & $\begin{array}{l}g_{\mathrm{e}}(\text { Lorentzian }) \\
\text { FWHM }_{\mathrm{e}}(\mathrm{mT})\end{array}$ & $\begin{array}{l}2.0049(5) \\
2.3(1)\end{array}$ & & $\begin{array}{l}2.0049(5) \\
1.8(1)\end{array}$ & & $\begin{array}{l}\text { cond. band tail } \\
\text { a-Si:H }\end{array}$ \\
\hline $\mathrm{h}$ & $\begin{array}{l}g_{\mathrm{h}}(\text { Gaussian }) \\
\text { FWHM }_{\mathrm{h}}(\mathrm{mT})\end{array}$ & & & $\begin{array}{l}2.011 \\
2.9\end{array}$ & $\begin{array}{l}2.011 \\
2.9\end{array}$ & $\begin{array}{l}\text { val. band tail } \\
\text { a-Si:H }\end{array}$ \\
\hline $\mathrm{P}$ & $\begin{array}{l}g_{\mathrm{P}}(\text { Gaussian }) \\
\text { FWHM }_{\mathrm{P}}(\mathrm{mT})\end{array}$ & $\begin{array}{l}2.003 \\
\text { hf split.: } \\
25 \mathrm{mT} \\
11.3\end{array}$ & & $\begin{array}{l}2.003 \\
\text { splitting: } \\
25 \mathrm{mT} \\
11.3\end{array}$ & & $\begin{array}{l}\text { phosph. donor } \\
\text { a-Si:H }\end{array}$ \\
\hline $\mathrm{sd}$ & $\begin{array}{l}g_{\text {sd }}(\text { Gaussian }) \\
\text { FWHM }_{\text {sd }}(\mathrm{mT})\end{array}$ & & $\begin{array}{l}1.9675(5) \\
2.5\end{array}$ & & & $\begin{array}{l}\text { shallow donor } \\
\mathrm{ZnO}\end{array}$ \\
\hline $\mathrm{db}$ & $\begin{array}{l}g_{\mathrm{db}}(\text { Lorentzian }) \\
\mathrm{FWHM}_{\mathrm{db}}(\mathrm{mT})\end{array}$ & & $\begin{array}{l}2.0045(5) \\
1.3(1)\end{array}$ & & & $\begin{array}{l}\text { dangling bond } \\
\mu \mathrm{c}-\mathrm{Si}: \mathrm{H}\end{array}$ \\
\hline $\mathrm{CH}$ & $\begin{array}{l}g_{\mathrm{CH}}(\text { asym. }) \\
\text { FWHM }_{\mathrm{CH}}(\mathrm{mT})\end{array}$ & & $\begin{array}{l}g_{\|}=2.15 \\
g_{\perp}=1.995 \\
5 \text { (Lorentz.) } \\
\Delta g_{\|}=0.2 \\
\Delta g_{\perp}=0.01\end{array}$ & & & $\begin{array}{l}\text { val. band tail } \\
\mu c-S i: H\end{array}$ \\
\hline
\end{tabular}

mw pulse leads to an increase of the hopping rate and thus can be considered to enhance the mobility in the hopping transport path. It has been shown that mobility modulations in thin-film silicon solar cells can strongly affect the conductivity, in particular at low temperatures $[43,44]$. In contrast to that, one would expect an initial quenching of the photocurrent $\left(\Delta\left|I_{\mathrm{ph}}\right|<0\right)$ for spin-dependent recombination or trapping. In this case, the conductivity is altered by a spin-resonant change of the charge carrier concentrations. Note that the abovementioned spindependent processes can be connected to other charge extraction limitations such as transport over energy barriers at contacts, recombination in a space charge region, space charge limited currents or trap assisted tunnelling through barriers. In such a case it is difficult to predict the sign of the photocurrent change since these processes can coexist and influence the current response of the device in a rather complex manner. However, irrespective of the spin-dependent transport limitation, theory predicts that the pEDMR transient shows a sign reversal when non-vanishing triplet transition probabilities are present $[14,18]$. To discriminate between various mechanisms, additional information is needed which we may obtain from the dynamics of the pEDMR signals as well as from coherent spin motion experiments.

\subsubsection{Analysis of $p E D M R$ transients}

To exploit in which way the different signals affect the charge transport in the device, we deconvoluted the pEDMR signals in time domain by taking the line parameters from the spectra shown in figure 6 (cf. table 2) as fixed parameters and their relative intensities as fit parameters. Thus, we were able to independently study the time behaviour of the spectrally overlapping signals. In this way we obtained the integrated peak intensities $A$ of all resonances. In order to cross-check the validity of the spectral fits (see figure 6$)$, the three lines at $g_{\mathrm{CE}}(\mathrm{FWHM}=$ $1.8 \mathrm{mT}), g_{\mathrm{e}}(\mathrm{FWHM}=1.8 \mathrm{mT})$ and $g_{\mathrm{h}}(\mathrm{FWHM}=2.9 \mathrm{mT})$ were taken into account for all samples.

Figure 8 depicts the time evolution of $A$ for samples A to D. From the time 
Figure 8. Time-dependent integrated peak intensities $A$ of the deconvoluted pEDMR signals of samples A to D. The deconvolution procedure was applied to the experimental data obtained with narrow magnetic field range (cf. figure 6). The $g$ values and line widths were assumed to be identical for all samples. Details of the deconvolution procedure are described in the text.

traces shown it is obvious that the three pEDMR signals $\mathrm{CE}$, e and $\mathrm{h}$ exhibit different dynamics. This indicates that none of the paramagnetic states associated with these resonances belong to the same spin-dependent process. In this case one would expect that both peak intensity and dynamics of two lines are correlated. Beside the different rise and fall times, all three signals show the same behaviour with respect to the observed sign of the mw induced effect (enhancing signal), except for the line at $g_{\mathrm{e}}$ in sample B which shows a photocurrent decrease after the mw excitation (quenching signal). The small dip at short times $(t<3 \mu \mathrm{s})$ of this quenching signal is a measurement and data processing artefact. Although the $g$ value of the quenching signal in sample B (see figure 6) seems indistinguishable from the $g$ value of signals at $g_{e}$ in sample A and C, we ascribe this quenching signal to a different paramagnetic state, namely dangling bond states in the intrinsic absorber layer. This argumentation is supported by the fact that we are able to resolve a small $g$ value shift between the enhancing lines of samples $\mathrm{A}$ and $\mathrm{C}$ as compared to the quenching signal in sample B. In addition, the quenching signal exhibits different dynamics and can easily be separated from the CE line in sample B by evaluating the time evolution of the spectra. Figure 9 shows a comparison between two spectra recorded at $t=2.2$ and $4 \mu$ s after the mw pulse excitation. One can clearly observe the quenching contribution at $t=4 \mu$ s which can easily be fitted separately. From this analysis we find $g_{\mathrm{db}}=2.0045(5)$ which slightly differs from $g_{\mathrm{e}}$ as it was found for samples A and C. Within the experimental uncertainty this agrees with the $g$ values reported for Si dangling bonds in $\mu \mathrm{c}-\mathrm{Si}: \mathrm{H}(g=2.0042$ 2.0058 depending on the deposition conditions) [5, 45]. Note that despite of the difference between $g_{\mathrm{db}}$ and $g_{\mathrm{e}}$, the temporal evolution of the quenching signal (figure 8 ) is reflected by the line at $g_{\mathrm{e}}=2.0049$ (deduced from the enhancing signal found in samples A and C, cf. figure 6). At present it is not clear why this quenching 
Figure 9. pEDMR spectrum of sample B recorded $4.0 \mu$ s after the mw pulse. Two lines with different $g$ values and opposite signs can clearly be distinguished (solid line: experiment; dashed line: result of a fit with two Lorentzian lines; dotted lines: both separate contributions to the fit). Note that the spectrum has been multiplied by a factor of two to allow for a better comparison with the spectrum recorded at $2.2 \mu \mathrm{s}$ which is also included in the plot.

signal is not also observed in samples A, C and D. Either this signal is associated with the specific interface of the intrinsic and the doped layers or the signal is simply masked by the strong signals related to the doped a-Si:H layers.

To evaluate the dynamics of the $25 \mathrm{mT}$ hf split lines that show up in the spectra of samples $\mathrm{A}$ and $\mathrm{C}$, the deconvolution procedure on these samples was additionally carried out using the pEDMR datasets with broad magnetic field range. Here, we assumed the same parameters as described before for the CE and e signals but, for the sake of simplicity, omitted the line at $g_{\mathrm{h}}$. The $\mathrm{P}$ signal was taken account of by assuming a pair of symmetric Gaussian lines with equal intensity that are split by $25 \mathrm{mT}$ and have their centre-of-gravity at $g_{\mathrm{P}}=2.003$. Figure 10 shows the results of this analysis.

While the time dependence of the CE and e signals resembles that given in figure 8 , we observe a clear correlation between the rise and fall times of the lines at $g_{\mathrm{e}}$ and $g_{\mathrm{P}}$ for both samples. For this to occur, both centres must either be involved in the same microscopic process or two different processes involving the two paramagnetic centres separately, but influence $I_{\mathrm{ph}}$ in a similar way [46]. Based on the experimental results we cannot unambiguously discriminate between both possibilities, but in any case the correlation between the pEDMR transients supports the interpretation that both signals stem from spin-dependent processes in the n-a-Si:H layer.

\subsubsection{Coherent spin motion}

Further information about charge transport mechanisms and the involvement of the defects assigned above may be obtained by analysing Rabi oscillations of the observer spins localised at the respective defect states. In particular, one can distinguish a spin-dependent transition (recombination or tunnelling) that involves only one paramagnetic centre - like direct capture recombination at silicon dan- 


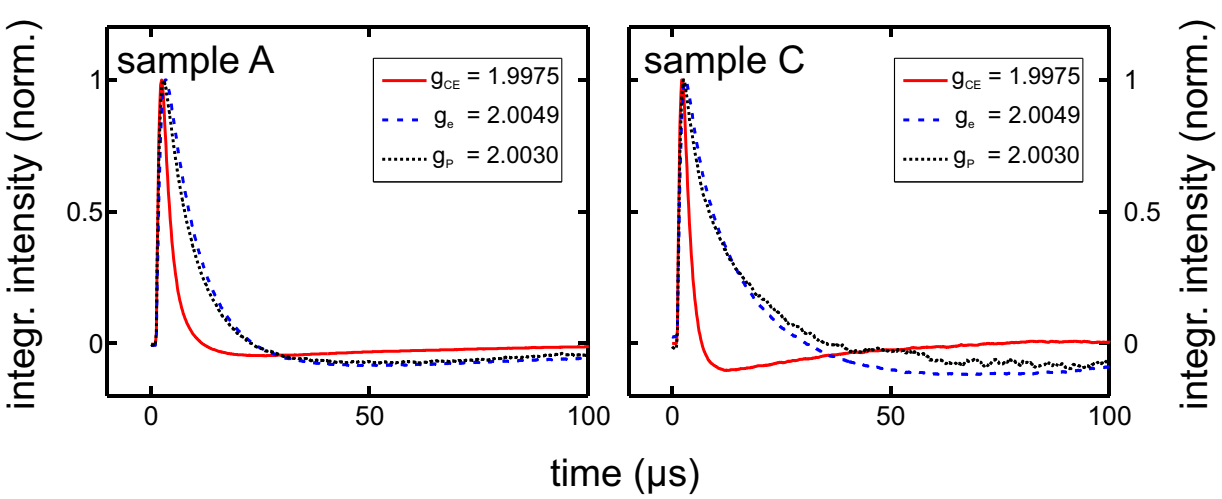

Figure 10. Integrated peak intensities for samples A and C (cells with a-Si:H n-layer). The deconvolution procedure performed using the two-dimensional pEDMR datasets with wide magnetic field range (cf. main plots in figure 6). The hyperfine signal that is visible in the respective spectra was taken into account by assuming a pair of Gaussian lines that are symmetric to $g_{\mathrm{P}}=2.003$. Note that the ordinate scale is normalised to facilitate the comparison of the dynamics.

gling bonds or a hopping process among tail states - from a process in which the spin-dependent transition takes place between two centres having different $g$ values $[39,40,46]$. In the latter case, one can address the spins of the charge carriers in both states separately by choosing the resonance condition according to the $g$ value of the respective spin. The frequency of the coherent spin motion is given by the Rabi frequency of the paramagnetic centre which is in resonance, provided that the driving field $\gamma B_{1}\left(B_{1}\right.$ denotes the amplitude of the mw magnetic field and $\gamma$ is the gyromagnetic ratio) is small compared to the Larmor separation $\delta \omega=\left(\omega_{a}-\omega_{b}\right)$ of the two respective centres $[47,48]$. Here, $\omega_{a}$ and $\omega_{b}$ are the Larmor frequencies of the two centres $a$ and $b$, respectively. On the contrary, when the Larmor separation of two participating centres is smaller than the driving field $\left(\delta \omega \ll \gamma B_{1}\right)$, theory predicts a Rabi frequency that is higher by a factor of two $[14,49,50]$.

To observe Rabi oscillations in pEDMR, the charge $Q(\tau)$ resulting from the integration of $\Delta\left|I_{\mathrm{ph}}\right|$ over several microseconds after the mw excitation is recorded as a function of the pulse length $\tau$ [15]. Figure 11 illustrates the charge response $Q(\tau)$ obtained on two different spectral positions (indicated by the $g$ values as given in the legend) for each sample. The insets show fast fourier transformations (FFT) of $Q(\tau)$ clearly indicating similar Rabi frequencies $(\Omega / 2 \pi=12-15 \mathrm{MHz})$ for all signals. Under the experimental conditions used in the experiment this corresponds to the Rabi frequencies we expect for a spin-dependent transition between weakly coupled spins that can be resolved in the pEDMR spectrum $\left(\delta \omega \gg \gamma B_{1}\right)$. It should be stressed that, unfortunately, the weak peak intensities of the broad lines (hf and $\mathrm{CH})$ do not allow us to determine their Rabi frequencies.

Assuming that all EDMR signals found in this study can be explained by the spin pair model [39], in which the relative spin orientation of both partners of a spin pair determines a microscopic transition rate, two spectrally distinguishable paramagnetic states are involved in each spin-dependent transition such as recombination or hopping process. When the spectral positions and/or line widths of these resonances differ significantly, which is true for the signals e and P in samples A and C, the frequency measured in the coherent spin motion experiment reflects the Rabi oscillation of the centre which is in resonance (e signal in this case) [14]. In the opposite case, when the spin pair consists of two paramagnetic centres with similar $g$ values and homogeneous broadening, only one line appears in the pEDMR spectrum. However, in this case it is impossible to manipulate only one spin partner without influencing the other (often referred to as the case of strong light-field coupling) [47, 48], and thus, one measures a Rabi frequency which is predicted to 
be higher by a factor of two as compared to the case before [14, 49, 50]. Only if the lines of both spins are strongly inhomogeneously broadened, the spectral overlap between individual spin packets of both lines may be small enough to form a weakly coupled spin pair.

All Rabi measurements presented here indicate spin-dependent transitions between paramagnetic centres which do not overlap in the EDMR spectra. This, however, raises the question which EDMR resonance line represents the respective recombination or hopping partner. For the EDMR signals which could be assigned to the n-a-Si:H layer, this puzzle may be answered referring to the transients of the EDMR signals shown in figure 6 . Both signals e and $\mathrm{P}$ have the same dynamics and their resonances in the pEDMR spectrum are clearly separated. Hence, they may constitute a weakly coupled spin pair, where the spin partners may be excited independently by the microwave pulse, resulting in the observed Rabi frequencies. This indicates a spin-dependent process between the two respective centres. Following the previous interpretation of the centres, we assign the spin-dependent process to hopping between band tail and phosphorous states in a-Si:H [46].

For the signals related to the $\mu \mathrm{c}-\mathrm{Si}: \mathrm{H}$ absorber layer this question remains an obstacle because the transients depicted in figure 8 exhibit completely different time constants. At present it is not clear how these paramagnetic centres are related to microscopic transport or recombination processes. A straightforward explanation of the observed Rabi behaviour can only be delivered if we assume that in the case of the signals $\mathrm{CE}, \mathrm{CH}$ and $\mathrm{db}$ the respective pair partners are not visible in the pEDMR spectra due to the fact that their lines are strongly inhomogeneously broadened or that the signals arise from a microscopic process involving more than 


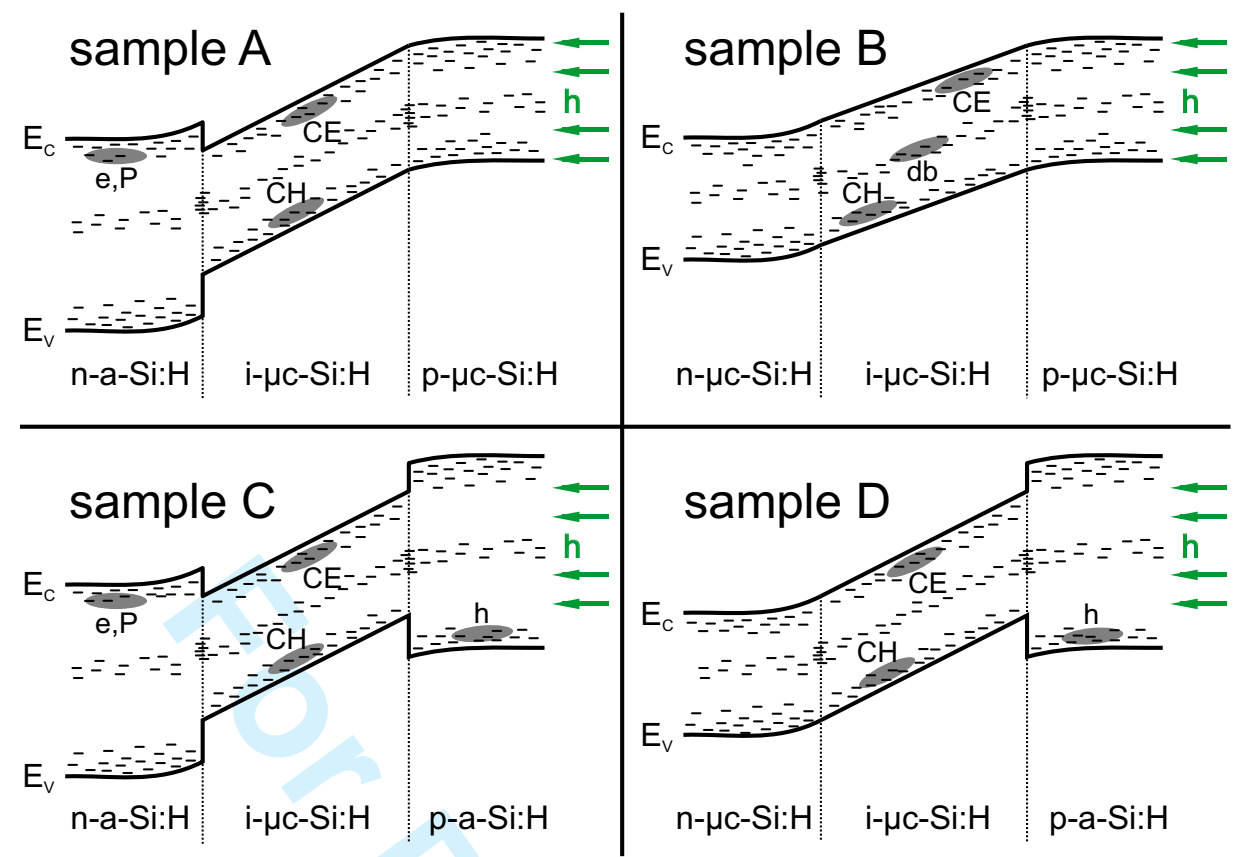

Figure 12. Sketch of energy-band diagrams at room temperature without illumination for samples A to D. The paramagnetic centres that were found to be involved in spin-dependent processes in the respective samples are shown schematically. Details can be found in the text.

two spins.

The paramagnetic centres that were identified by the pEDMR analyses described above are summarised in figure 12 .

\section{Conclusion and outlook}

The preceding pEDMR study demonstrates that an identification of paramagnetic defect states and charge transport processes may be obtained in completely processed pin solar cells. The complexity of the problem to assign the defect states to materials and interfaces in a multi-layer solar cell made a systematic alternation of the morphology of the highly doped n- and p-layers mandatory. We could show that by combining the information obtained from pEDMR spectra and from the time evolution of the spin-dependent photocurrent transients the spectral resolution may be significantly enhanced. As a result, valuable additional information about transport mechanisms could be obtained as compared to conventional ESR and EDMR investigations.

Thereby we succeeded in identifying electron hopping processes between conduction band tail states and phosphorus donor states in the n-a-Si:H layer. Similarly, spin-dependent processes involving holes in valence band tail states in the p-a-Si:H layer could be observed. Presumably, these states are involved in a hopping or tunnelling process, however, the details of these mechanisms are at present unclear. Paramagnetic states in the intrinsic $\mu \mathrm{c}-\mathrm{Si}: \mathrm{H}$ absorber, which was present in all samples, give rise to pronounced resonances. A strong signal related to localised electrons in the conduction band tail (CE) was observed in the i- $\mu c-S i: H$ layer. In addition, we found a signal which we assign to holes localised in the valence band tail $(\mathrm{CH})$. These states give rise to an asymmetric and broad line that could be simulated assuming a strongly asymmetric $g$ tensor and asymmetric line broadening. Signals related to dangling bonds in the $\mu \mathrm{c}-\mathrm{Si}: \mathrm{H}$ absorber could only be observed for the sample with microcrystalline n- and p-layers. This is of particular importance for prospective studies which address the investigation of spin- 


\section{garino \\ Philosophical}

dependent recombination via dangling bonds in the absorber layer created though the Staebler-Wronski effect [51]. Our findings indicate that the strong EDMR signals caused by tail states in the highly doped layers can be suppressed by using microcrystalline $\mathrm{n}$ - and p-layers.

Besides this, we were able to detect a pEDMR signal at $g=1.9675(5)$ in the $\mu \mathrm{c}-\mathrm{Si}: \mathrm{H}$ pin solar cell with p- and n-doped $\mu \mathrm{c}-\mathrm{Si}: \mathrm{H}$ layers which we associate with shallow donor states in the $\mathrm{Al}$-doped $\mathrm{ZnO}$. Here, more work is needed on thin $\mathrm{ZnO}$ layers and devices, in which the $\mathrm{ZnO}$ is replaced by other transparent conducting oxides, to further investigate the microscopic nature of this resonance.

Coherent spin motion measurements revealed the signature of spin-dependent transitions in which both spins of the respective spin pair can be addressed separately. In the case of the hopping signals originating from the n-a-Si:H layer, this is in agreement with the pEDMR spectra, provided that we indeed observe a hopping process from a phosphorus donor state to a band tail state or vice versa. In the case of the resonances that stem from the p-a-Si:H and i- $\mu \mathrm{c}-\mathrm{Si}: \mathrm{H}$ layers, no such correlation between two signals could be found in the pEDMR spectra and transients. This indicates that either the missing spin partners cannot be observed in the spectra or that the spin pair model cannot account for the underlying microscopic mechanisms. Due to its strong inhomogeneity, spin-dependent processes through the $\mathrm{CH}$ states could serve as the missing link in the interpretation of the Rabi oscillations. To connect the $\mathrm{CH}$ states to a specific process, the dynamic behaviour of this line has to be studied in greater detail which, however, is difficult in view of the relatively small signal amplitude.

The present study was performed at a temperature of $T=10 \mathrm{~K}$ where charge transport dynamics are distinctively different from the dominating mechanisms at room temperature, i.e. under operating conditions of solar cells. However, the identification of defect states and transport mechanisms at low temperatures constitutes the basis for studies focusing on the temperature dependence of these mechanisms. It shall be mentioned here that due to instrumental improvements in our lab, pEDMR experiments are no longer restricted to the low-temperature range. Benefiting from these improvements we recently succeeded in performing pEDMR measurements (photocurrent relaxation as well as coherent experiments) at room temperature, which will pave the way to study spin-dependent transport in multi layer solar cells at room temperature in the near future.

Further on, the identification of defects and transport mechanisms obtained in the present work is an inevitable step towards multi-frequency and multi-resonance pEDMR studies of individual paramagnetic centres. Here, our aim is to combine the sensitivity and specificity to charge transport mechanisms of pEDMR with the spectral resolution of advanced EPR techniques [52] to shed light on the functionstructure relationship of individual defect states and transport pathways.

\section{Acknowledgements}

We are grateful to M.A. Gluba and O. Astakhov for helpful discussions. This work was partially funded by the German Federal Ministry of Education and Research (BMBF network project EPR-Solar 03SF0328A).

\section{References}

[1] W.E. Spear and P.G. Lecomber, Solid State Commun. 17 (1975) p.1193-1196.

[2] J. Müller, F. Finger, R. Carius and H. Wagner, Phys. Rev. B 60 (1999) p.11666-11677.

[3] W. Fuhs, J. Non-Cryst. Solids 354 (2008) p.2067-2078. 
[4] T. Umeda, S. Yamasaki, J. Isoya and K. Tanaka, Phys. Rev. B 59 (1999) p.4849-4857.

[5] F. Finger, L.B. Neto, R. Carius, T. Dylla and S. Klein, phys. stat. sol. (c) 1 (2004) p.1248-1254.

[6] M. Stutzmann, M.S. Brandt and M.W. Bayerl, J. Non-Cryst. Solids 266 (2000) p.1-22.

[7] I. Solomon, D. Biegelsen and J.C. Knights, Solid State Commun. 22 (1977) p.505-508.

[8] E.A. Schiff, AIP Conf. Proc. 73 (1981) p.233-237.

[9] R.A. Street, Philos. Mag. B 46 (1982) p.273-278.

10] I. Solomon, Solid State Commun. 20 (1976) p.215-217.

[11] F.C. Rong, G.J. Gerardi, W.R. Buchwald, E.H. Poindexter, M.T. Umlor, D.J. Keeble and W.L. Warren, Appl. Phys. Lett. 60 (1992) p.610-612.

[12] F.H.L. Koppens, C. Buizert, K.J. Tielrooij, I.T. Vink, K.C. Nowack, T. Meunier, L.P. Kouwenhoven and L.M.K. Vandersypen, Nature 442 (2006) p.766-771.

[13] D.R. McCamey, H. Huebl, M.S. Brandt, W.D. Hutchison, J.C. McCallum, R.G. Clark and A.R. Hamilton, Appl. Phys. Lett. 89 (2006) p.182115.

[14] C. Boehme and K. Lips, Phys. Rev. B 68 (2003) p.245105.

[15] C. Boehme and K. Lips, Phys. Rev. Lett. 91 (2003) p.246603.

[16] J. Behrends, A. Schnegg, C. Boehme, S. Haas, H. Stiebig, F. Finger, B. Rech and K. Lips, J. NonCryst. Solids 354 (2008) p.2411-2415.

17] Y. Mai, S. Klein, R. Carius, H. Stiebig, X. Geng and F. Finger, Appl. Phys. Lett. 87 (2005) p.073503.

[18] C. Boehme and K. Lips, The investigation of charge carrier recombination and hopping transport with pulsed electrically detected magnetic resonance techniques, in Charge transport in disordered solids with applications in electronics, in Charge transport in disordered solids with applications in electronics, ed. S. BaranovskiS. Baranovski ed., Wiley, Chichester, England ; Hoboken, NJ, 2006.

[19] S. Hasegawa, S. Narikawa and Y. Kurata, Philos. Mag. B 48 (1983) p.431-447.

[20] F. Finger, J. Muller, C. Malten and H. Wagner, Philos. Mag. B 77 (1998) p.805-830.

[21] M. Kondo, T. Nishimiya, K. Saito and A. Matsuda, J. Non-Cryst. Solids 230 (1998) p.1031-1035.

[22] P. Kanschat, K. Lips and W. Fuhs, J. Non-Cryst. Solids 266 (2000) p.524-528.

[23] M.M. deLima, P.C. Taylor, S. Morrison, A. LeGeune and F.C. Marques, Phys. Rev. B 65 (2002) p. 235324 .

[24] M. Stutzmann and R.A. Street, Phys. Rev. Lett. 54 (1985) p.1836-1839.

[25] K. Lips, S. Schütte and W. Fuhs, Philos. Mag. B 65 (1992) p.945-959.

[26] C. Boehme, J. Behrends, K.V. Maydell, M. Schmidt and K. Lips, J. Non-Cryst. Solids 352 (2006) p.1113-1116.

[27] K. Lips, P. Kanschat and W. Fuhs, Sol. Energy Mat. Sol. Cells 78 (2003) p.513-541.

[28] M.S. Brandt and M. Stutzmann, Phys. Rev. B 43 (1991) p.5184-5187.

[29] H. Dersch, J. Stuke and J. Beichler, phys. stat. sol. (b) 107 (1981) p.307-317.

[30] H. Dersch, L. Schweitzer and J. Stuke, Phys. Rev. B 28 (1983) p.4678-4684.

[31] S. Depinna, B.C. Cavenett, I.G. Austin, T.M. Searle, M.J. Thompson, J. Allison and P.G. Lecomber, Philos. Mag. B 46 (1982) p.473-500.

[32] F. Boulitrop, Phys. Rev. B 28 (1983) p.6192-6208.

[33] K. Morigaki, D.J. Dunstan, B.C. Cavenett, P. Dawson, J.E. Nicholls, S. Nitta and K. Shimakawa, Solid State Commun. 26 (1978) p.981-985.

[34] S. Stoll and A. Schweiger, J. Magn. Res. 178 (2006) p.42-55.

[35] M. Stutzmann, D.K. Biegelsen and R.A. Street, Phys. Rev. B 35 (1987) p.5666-5701.

[36] T. Umeda, S. Yamasaki, J. Isoya and K. Tanaka, Phys. Rev. B 62 (2000) p.15702-15710.

[37] S.B. Orlinskii, J. Schmidt, P.G. Baranov, V. Lorrmann, I. Riedel, D. Rauh and V. Dyakonov, Phys. Rev. B 77 (2008) p.115334.

[38] D.M. Hofmann, A. Hofstaetter, F. Leiter, H.J. Zhou, F. Henecker, B.K. Meyer, S.B. Orlinskii, J. Schmidt and P.G. Baranov, Phys. Rev. Lett. 88 (2002) p.045504.

[39] D. Kaplan, I. Solomon and N.F. Mott, Journ. de Phys. Lettr. 39 (1978) p.L51-L54.

[40] F.C. Rong, W.R. Buchwald, E.H. Poindexter, W.L. Warren and D.J. Keeble, Solid-State Electron. 34 (1991) p.835-841.

[41] K. Lips, C. Boehme and W. Fuhs, IEE Proc. - Circ. Dev. Syst. 150 (2003) p.309-315.

[42] T. Brammer, H. Stiebig and K. Lips, Appl. Phys. Lett. 85 (2004) p.1625-1626.

[43] J.J. Liang, E.A. Schiff, S. Guha, B.J. Yan and J. Yang, Appl. Phys. Lett. 88 (2006) p.063512.

[44] E.A. Schiff, Sol. Energy Mat. Sol. Cells 78 (2003) p.567-595.

[45] O. Astakhov, R. Carius, Y. Petrusenko, V. Borysenk, D. Barankov and F. Finger, phys. stat. sol. (RRL) 1 (2007) p.R77-R79.

[46] C. Boehme and K. Lips, phys. stat. sol. (c) 1 (2004) p.1255-1274.

[47] V. Rajevac, C. Boehme, C. Michel, A. Gliesche, K. Lips, S.D. Baranovskii and P. Thomas, Phys. Rev. B 74 (2006) p.245206.

[48] A. Gliesche, C. Michel, V. Rajevac, K. Lips, S.D. Baranovskii, F. Gebhard and C. Boehme, Phys. Rev. B 77 (2008) p.245206.

[49] B.M. Tadjikov, A.V. Astashkin and Y. Sakaguchi, Chem. Phys. Lett. 283 (1998) p.179-186.

[50] Y. Araki, K. Maeda and H. Murai, Chem. Phys. Lett. 332 (2000) p.515-520.

51] D.L. Staebler and C.R. Wronski, Appl. Phys. Lett. 31 (1977) p.292-294.

[52] A. Schweiger and G. Jeschke Principles of pulse electron paramagnetic resonance, Oxford University Press, Oxford, UK ; New York, 2001. 